\title{
Mucinous adenocarcinoma arising from chronic perianal fistula
}

\begin{abstract}
Perianal mucinous adenocarcinoma is a rare tumour which may be associated with longstanding chronic perianal sepsis. Early diagnosis is challenging and is based on a high index of clinical suspicion and specific histological features. Definitive treatment is surgical, in the form of an abdomino-perineal resection. We hereby describe a case of a perianal mucinous adenocarcinoma arising from long-standing recurrent perianal fistula and complement this with a brief review of the literature pertaining in particular to the management of this condition.
\end{abstract}

Keyword: Mucinous adenocarcinoma; Perianal fistula; Perianal sepis 